09

\title{
Получение униполярных импульсов в дальней зоне источника
}

\author{
(c) М.В. Архипов ${ }^{1}$, Р.М. Архипов ${ }^{1}$, Н.Н. Розанов ${ }^{2}$ \\ ${ }^{1}$ Санкт-Петербургский государственный университет, \\ 199034 Санкт-Петербург, Россия \\ ${ }^{2}$ ФТИ им. А.Ф. Иоффре, \\ 194021 Санкт-Петербург, Россия \\ e-mail: m.arkhipov@spbu.ru, arkhipovrostislav@gmail.com, nnrosanov@mail.ru
}

Поступила в редакцию 27.05.2021 г.

В окончательной редакции 27.05.2021 r.

Принята к публикации 07.06.2021 г.

\begin{abstract}
При распространении импульсов негармонической формы в пустом пространстве зависимость напряженности поля от времени изменяется. В дальней зоне или при фокусировке излучения в точке фокуса напряженность поля становится пропорциональной производной по времени от поля источника. Если исходное поле униполярно, то дифференцирование приведет к потере униполярности. На основании этих рассуждений обычно делают вывод о крайне ограниченном практическом использовании излучения униполярных источников, поскольку всякое распространение и фокусировка подобного излучения приведут к потере униполярности. Однако это ограничение не влияет на ситуации, когда зависимость поля от времени имеет специальный вид. Приведены условия, при которых получение униполярных импульсов в дальней зоне возможно.
\end{abstract}

Ключевые слова: униполярные импульсы, предельно короткие импульсы, дальняя зона, дифракция, фокусировка.

DOI: $10.21883 /$ OS.2021.09.51345.2342-21

Несмотря на принципиальную возможность существования импульсов униполярного излучения в оптическом и смежных диапазонах, до недавнего времени интерес к получению и применению подобного излучения отсутствовал. По мере сокращения длительностей импульсов, переходу от фемто- к аттосекундному диапазону их длительностей встал вопрос о получении субцикловых импульсов, содержащих менее одного периода колебаний поля $[1,2]$. И стали обсуждаться вопросы, связанные с униполярностью и электрической площадью импульсов. Рассмотрение этих вопросов проведено в обзорах $[3,4]$ и монографии [5].

Практическая ценность униполярных импульсов в отличие от привычных биполярных импульсов, содержащих несколько циклов колебаний, видится в их способности однонаправленного воздействия на заряды, что позволяет, например, за более короткие времена нерезонансно возбуждать/девозбуждать квантовые системы [1,6-8] и ускорять свободные заряды [9], см. также и другие применения в обзоре [4] и цитируемой в нем литературе. Однако известно, что при распространении униполярных импульсов в пустом пространстве или их фокусировке происходит потеря униполярности [10-12].

Этот факт часто используется как аргумент в дискуссиях, показывающий малую практическую ценность работ по получению таких импульсов. Невозможность сохранить униполярность при удалении от источника, а также невозможность сконцентрировать излучение в малой области пространства для реализации высокой пиковой амплитуды поля ставит под вопрос перспективы применения униполярных импульсов. Соответственно целью настоящей работы является иллюстрация возможности получения униполярных импульсов в дальней зоне источника.

Отметим сначала два момента. Во-первых, электрическая площадь излучения зарядов в вакууме обращается в нуль, если и только если во всех точках пространства интегральная по времени плотность заряда равна нулю [13].

Во-вторых, строгая униполярность не является необходимым фактором для реализации тех преимуществ, которые дает полная униполярность перед биполярным импульсом. Наличие у импульса длинного фронта с противоположным направлением напряженности поля, разнесение двух униполярных импульсов с разной полярностью во времени позволяет применять такое излучение так же эффективно, как униполярное [7,8]. Этот вывод качественно подтверждается экспериментальными исследованиями, проведенными в работе [1], в которой показано, что субцикловые импульсы, содержащие длинный фронт противоположной полярности, более эффективно возбуждают атомную систему, чем одноцикловые импульсы.

Получить излучение, содержащее либо один всплеск напряженности поля с большой амплитудой и малой длительностью с длинным фронтом малой амплитуды либо два разнесенных во времени униполярных импульса противоположной полярности в дальней зоне от источника (или при фокусировке) можно, если источник излучения (ближняя зона) имеет специальный времен- 

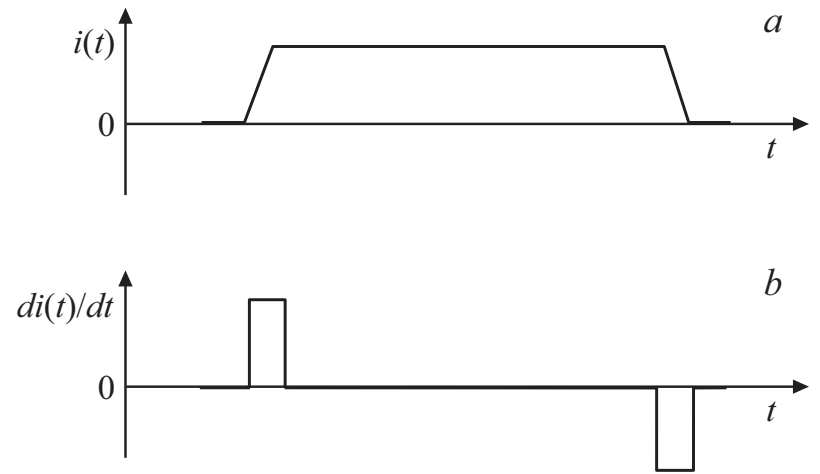

Импульс тока трапецеидальной формы $(a)$ и его производная $(b)$.

ной профиль. Подобные импульсы имеют применение в радиолокации и радиосвязи несинусоидальных волн [14].

Идею получения несинусоидальных униполярных волн иллюстрирует рисунок. Здесь ток в антенне передатчика радиолокатора имеет трапецеидальную зависимость от времени (рис., $a$ ). Напряженность электрического поля $\mathbf{E}$ излучения, порождаемого током с плотностью током $\mathbf{j}$, описывается волновым уравнением, решение которого (запаздывающие потенциалы) в дальней зоне (на расстоянии $R_{0}$ от излучателей) имеет вид [15]

$$
\mathbf{E}=-\left.\frac{1}{c^{2} R_{0}} \int \frac{\partial \mathbf{j}}{\partial t}\right|_{t-R / c} d V .
$$

При этом в ближней зоне, в которой дифракция проявляется слабо и оправдано плосковолновое рассмотрение, напряженность генерируемого источником поля пропорциональна току $[4,16,17]$ :

$$
E(z, t)=-\frac{2 \pi}{c} \int_{z_{1}}^{z_{2}} j\left(z^{\prime}, t-\frac{\left|z-z^{\prime}\right|}{c}\right) d z^{\prime} .
$$

Здесь интегрирование ведется по слою, который заключен между точками с координатами $z_{1}$ и $z_{2}$.

Согласно (1), в дальней зоне или при фокусировке напряженность поля будет пропорциональна производной тока. Тем самым поле в дальней зоне будет иметь вид двух разнесенных во времени униполярных импульсов с противоположными направлениями напряженности поля (рис. $b$ ). Их длительность будет равна длительности переднего и заднего фронтов трапецеидального импульса. В обзоре [4] также был дан пример формирования поля ускорившего заряда, затем движущегося равномерно и замедляющегося до полной остановки, что приводит к появлению двух коротких импульсов в дальней зоне. Если бы заряд не остановился, то мы получили бы строго униполярный импульс в дальней зоне и при фокусировке. Физическая ситуация аналогична изображенной на рисунке. Ряд других предложений по получению униполярных импульсов прямоугольной и трапецеидальной форм изложен в [18-21].
Обратим внимание, что при получении очень коротких импульсов при облучении лазерным излучением различных мишеней [22-25] источником излучения является ускоренное направленное движение электронов, т.е. импульс тока в заданном условиями эксперимента направлении. Если он имеет крутой фронт и более медленное затухание, то в дальней зоне или при фокусировке мы получим субцикловый импульс с длинным фронтом противоположной полярности.

Таким образом, если требуется достижение униполярных свойств излучения при фокусировке или распространении униполярного излучения в пустом пространстве на значительные расстояния, то это решается за счет реализации соответствующей формы импульса в ближней зоне источника. Напомним также, что униполярные импульсы могут транспортироваться в коаксиальных волноводах [26].

\section{Финансирование работы}

Работа выполнена при финансовой поддержке РФФИ в рамках научных проектов 20-32-70049 (проблема получения униполярных импульсов) и 19-02-00312 (проблема изменения электрической площади при распространении предельно коротких импульсов).

\section{Конфликт интересов}

Авторы заявляют, что у них нет конфликта интересов.

\section{Список литературы}

[1] Hassan M.T., Luu T.T., Moulet A., Raskazovskaya O., Zhokhov P., Garg M., Karpowicz N., Zheltikov A.M., Pervak V., Krausz F., Goulielmakis E. // Nature. 2016. V. 530. P. 66.

[2] Rossi G.M., Mainz R.E., Yang Y., Scheiba F., Silva-Toledo M.A., Chia S.H., Keathley P.D., Fang S., Mücke O.D., Manzoni C., Cerullo G., Cirmi G., Kärtner F.X. // Nature Photonics. 2020. V. 14 (10). P. 629-635.

[3] Розанов Н.Н., Архипов Р.М., Архипов М.В. // УФН. 2018. T. 188. № 12. C. 1347; Rosanov N.N., Arkhipov R.M., Arkhipov M.V. // Phys. Usp. 2018. V. 61. N 12. P. 1227.

[4] Архипов Р.М., Архипов М.В., Розанов Н.Н. // Квант. электрон. 2020. Т. 50. № 9. С. 801-815; Arkhipov R.M., Arkhipov M.V., Rosanov N.N. // Quant. Electron. 2020. V. 50. N 9. P. $801-815$.

[5] Розанов Н.Н. Диссипативные оптические и родственные солитоны. М.: Физматлит, 2021.

[6] Arkhipov R.M., Arkhipov M.V., Babushkin I., Demircan A., Morgner U., Rosanov N.N. // Opt. Lett. 2019. V. 44. N 5. P. 1202.

[7] Архипов Р.М., Архипов М.В., Пахомов А.В., Розанов Н.Н. // Опт. и спектр. 2020. Т. 128. В. 1. C. 106-109; Arkhipov R.M., Arkhipov M.V., Pakhomov A.V., Rosanov N.N. // Opt. Spectrosc. 2020. V. 128. N 1. P. 102-105. 
[8] Arkhipov R., Pakhomov A., Arkhipov M., Demircan A., Morgner U., Rosanov N., Babushkin I. // Opt. Express. 2020. V. 28. N 11. P. $17020-17034$.

[9] Розанов Н.Н., Высотина Н.В. // ЖЭТФ. 2020. Т. 157. № 1. C. 63-66; Rosanov N.N., Vysotina N.V. // JETP. 2020. V. 130. N 1. P. $52-55$.

[10] Розанов Н.Н. // Опт. и спектр. 2003. Т. 95. С. 318; Rozanov N.N. // Opt. Spectrosc. 2003. V. 95. P. 299.

[11] You D., Bucksbaum P.H. // J. Opt. Soc. Am. B. 1997. V. 14. P. 1651.

[12] Kaplan A.E. // J. Opt. Soc. Am. B. 1998. V. 15. P. 951.

[13] Розанов Н.Н. // Опт. и спектр. 2020. Т. 128. С. 95; Rosanov N.N. // Opt. Spectrosc. 2020. V. 128. P. 92.

[14] Хармут Х.Ф. Несинусоидальные волны в радиолокации и радиосвязи. М.: Радио и связь, 1985; Harmuth H.F. Nonsinusoidal Waves for Radar and Radio Communication. NY: Acad. Press, 1981.

[15] Ландау Л.Д., Лифини Е.М. Теория поля. М.: Гл. ред. физ.-мат. лит., 1988; Landau L.D., Lifshitz E.M. The Classical Theory of Fields. Butterworth-Heinemann: Oxford, 1975.

[16] Arkhipov M.V., Arkhipov R.M., Pakhomov A.V., Babushkin I.V., Demircan A., Morgner U., Rosanov N.N. // Opt. Lett. 2017. V. 42. P. 2189.

[17] Пахомов А.В., Архипов Р.М., Архипов М.В., Бабушкин И., Розанов Н.Н. // Опт. и спектр. 2017. Т. 123. С. 901; Pakhomov A.V., Arkhipov R.M., Arkhipov M.V., Babushkin I., Rosanov N.N. // Opt. Spectrosc. 2017. V. 123. P. 913.

[18] Arkhipov R.M., Arkhipov M.V., Belov P.A., Tolmachev Yu.A., Babushkin I. // Las. Phys. Lett. 2016. V. 13. N 4. P. 046001.

[19] Arkhipov R.M., Pakhomov A.V., Babushkin I.V., Arkhipov M.V., Tolmachev Yu.A., Rosanov N.N. // J. Opt. Soc. Am. B. 2016. V. 33. N 12. P. 2518-2524.

[20] Pakhomov A.V., Arkhipov R.M., Babushkin I.V., Arkhipov M.V., Tolmachev Yu.A., Rosanov N.N. // Phys. Rev. A. 2017. V. 95. N 1. P. 013804.

[21] Pakhomov A.V., Arkhipov R.M., Arkhipov M.V., Demircan A., Morgner U., Rosanov N.N., Babushkin I. // Sci. Rep. 2019. V. 9. Article Number 7444.

[22] Gao Y., Drake T., Chen Z., De Camp M.F. // Opt. Lett. 2008. V. 33. P. 2776.

[23] Xu J., Shen B., Zhang X., Shi Y., Ji L., Zhang L., Xu T., Wang W., Zhao X., Xu Z. // Sci. Rep. 2018. V. 8. P. 2669.

[24] Fülöp J.A., Tzortzakis S., Kampfrath T. // Adv. Opt. Mater. 2020. V. 8. P. 1900681.

[25] Shou Y., Hu R., Gong Z., Yu J., Chen J., Mourou G., Yan X., Ma W. // New J. Phys. 2021. V. 23. N 5. P. 053003.

[26] Розанов Н.Н. // Опт. и спектр. 2019. Т. 127. С. 960; Rosanov N.N. // Opt. Spectrosc. 2019. V. 127. P. 1050. 